\title{
A single institutional experience with central lung stereotactic body radiation therapy demonstrating encouraging results with increased inter-fraction time
}

\author{
Roman O. Kowalchuk $^{1{ }^{*}}$, Michael R. Waters ${ }^{1 * *}$, K. Martin Richardson ${ }^{1}$, Kelly M. Spencer ${ }^{1}$, \\ James M. Larner ${ }^{2}$, Charles R. Kersh ${ }^{1}$ \\ ${ }^{1}$ Radiosurgery Center, University of Virginia/Riverside, Newport News, VA, USA; ${ }^{2}$ Department of Radiation Oncology, University of Virginia, \\ Charlottesville, VA, USA \\ Contributions: (I) Conception and design: RO Kowalchuk, CR Kersh; (II) Administrative support: CR Kersh, JM Larner; (III) Provision of study \\ materials or patients: CR Kersh, KM Richardson, KM Spencer; (IV) Collection and assembly of data: RO Kowalchuk, CR Kersh; (V) Data analysis \\ and interpretation: RO Kowalchuk, MR Waters, CR Kersh; (VI) Manuscript writing: All authors; (VII) Final approval of manuscript: All authors. \\ Correspondence to: Roman O. Kowalchuk, MD. Radiosurgery Center, University of Virginia/Riverside, 500 J Clyde Morris Blvd, Newport News, VA \\ 23601, USA. Email addresses: kowalchuk.roman@mayo.edu; okowal17@gmail.com.
}

Background: Stereotactic body radiation therapy (SBRT) is an effective treatment modality for non-small cell lung cancer (NSCLC); however, there are concerns regarding potential toxicity for centrally located tumors.

Methods: This retrospective study considered patients with SBRT for central lung NSCLC (defined as a tumor within $2 \mathrm{~cm}$ of any mediastinal critical structure). The institutional protocol was that patients with central tumors received SBRT less frequently than daily—-generally once or twice weekly.

Results: A total of 115 patients with 148 lesions were treated with SBRT to a median 45 [5-60] Gy in 4 [1-5] fractions over a median 5.3 [0-18] days. Many patients treated with this method presented with advanced disease: 58 treatments involved nodal targets, and 42 had stage 3 disease. $52 \%$ of patients had chronic obstructive pulmonary disease (COPD), and only 49\% had a biopsy, often due to concerns regarding other medical comorbidities. Rates of prior chemotherapy, thoracic surgery, and thoracic radiotherapy were $32 \%$, $21 \%$, and $49 \%$, respectively. Via the Kaplan-Meier method, 2-year overall survival was $65 \%$, and 2 -year local control was $77 \%$. Two-year local-progression free survival was $53 \%$, and 2 -year progression-survival was $48 \%$. Treatments for stage 3 disease had an impressive $82 \%$-year local control that was comparable to early stage treatments. Patients with stage 3 disease had a 2 -year overall survival of $59 \%$, which trended towards decreased overall survival compared to early stage patients. There were 13 grade 1 (9\%) and 14 grade 2 (9\%) toxicities. There were no reported grade $\geq 3$ acute or late toxicities and only 3 cases of pneumonitis.

Conclusions: Our series demonstrates encouraging local control with low rates of toxicity for central lung SBRT, including many stage 3 patients. This may be the result of the relatively large inter-fraction interval. This interval may allow for greater tumor effects (such as reoxygenation) and improved tolerance from normal tissues.

Keywords: Dose fractionation; non-small cell lung cancer (NSCLC); patient selection; stereotactic body radiation therapy (SBRT); treatment outcome

\footnotetext{
$\wedge$ ORCID: 0000-0001-6142-6310.

* Current address: Mayo Clinic, Department of Radiation Oncology, 200 1st St SW, Rochester, MN, 55905, USA.

** Current address: Washington University in St. Louis, Department of Radiation Oncology, 660 S. Euclid Ave, St. Louis, MO, 63110, USA.
} 
Submitted Aug 13, 2020. Accepted for publication Oct 25, 2020.

doi: $10.21037 /$ jtd-20-2659

View this article at: http://dx.doi.org/10.21037/jtd-20-2659

\section{Introduction}

Stereotactic body radiation therapy (SBRT), also known as stereotactic ablative radiotherapy (SABR), delivers high doses of radiation in relatively few treatment fractions. Studies in non-small cell lung cancer (NSCLC) have demonstrated high rates of local control and low toxicity, allowing for its use in patients with significant medical comorbidities (1). As the refinement of patient selection continues, patients who are surgical candidates have also been considered, and population studies have associated the increasing utility of SBRT for elderly patients and improved overall survival $(2,3)$. Though SBRT has been favorably compared to conventional fractionation in early stage lung cancer, the dose-limiting toxicity, radiation pneumonitis, continues to affect patients after $9-28 \%$ of cases $(4,5)$.

Centrally located NSCLC carries the potential for increased rates of toxicity, largely secondary to the proximity to key central structures. Though varied definitions exist, most analyses define a central tumor as a lesion within 2 $\mathrm{cm}$ in all directions of any mediastinal critical structure (6). RTOG 0813 found a $7.2 \%$ rate of dose-limiting toxicity (grade $\geq 3$ ) with a maximum tolerated dose of 12.0 Gy/ fraction, but other studies have demonstrated lower rates of toxicity with central lung SBRT $(7,8)$. Two papers, including the prospective phase II trial from Bral et al., noted a correlation between local control and tumor size, which could subsequently affect dose delivery and toxicity $(9,10)$. These results have prompted some providers to increase the number of fractions for dose delivery up to 8 treatments (11). Notably, survival outcomes appear to be comparable between early stage NSCLC, regardless of central or peripheral location (12).

Further questions have arisen regarding "ultracentral" and hilar SBRT. "Ultra-central" tumors are generally defined as those with gross tumor volume (GTV) directly abutting the central airway. While some studies have shown encouraging outcomes, careful patient selection is recommended until large prospective trials are completed (13). A key concern emerges regarding prioritization of target coverage or organs at risk (14). Treatments for paramediastinal and hilar targets similarly challenge physicians (15). Hilar radiotherapy has been discussed in only a few studies, the largest of which considered 40 patients with hilar/mediastinal SBRT, finding any grade $\geq 3$ toxicity in 4 patients (10\%) (16-18).

This study seeks to analyze a large patient cohort treated with SBRT for central lung NSCLC and present outcomes of overall survival, progression-free survival, and localprogression free survival. This group consists of many treatments with uncommon and potentially high-risk features, such as node-positive disease, unbiopsied tumors, recurrent cancers, and stage 3 malignancies, and the authors seek to demonstrate that increased inter-fraction time may reduce the risk of side effects, like the PATRIOT trial in prostate cancer SBRT $(19,20)$. We present the following article in accordance with the STROBE reporting checklist (available at http://dx.doi.org/10.21037/jtd-20-2659).

\section{Methods}

\section{Patients}

Patients were considered if they received SBRT for a central lung tumor diagnosed as or empirically treated as NSCLC. Patients were treated from 2007-March 2018. A central lung cancer was defined as a tumor within $2 \mathrm{~cm}$ in all directions of any mediastinal critical structure. Exclusion criteria included a lack of clinical or imaging follow-up, a different primary tumor, or a peripheral location. Patients were not excluded from the study based on clinical stage, hilar or mediastinal location (grouped as "nodal" for the analysis), or a lack of biopsy. Patients were also not excluded from the study if they had a documented death but a lack of clinical or imaging follow-up. In such a case, the patient would be included in the survival analysis but not in the assessment of local control. Patient characteristics and outcomes were recorded via retrospective chart review, and this approach was exempt by the institutional review board due to the retrospective nature of the study and the establishment of a previous protocol for conducting retrospective studies. The initial patient set was developed to include all patients treated from 2007 through 2014 who met the above criteria. This resulted in an initial set of 105 lesions treated in 85 patients. A separate review of all 
treated SBRT cases from 2007 through 2018 was performed to additionally include all patients with treatments targeting the hilar or mediastinal regions. This resulted in the addition of 30 patients with 43 treated lesions to the dataset. This study was conducted in accordance with the Declaration of Helsinki (as revised in 2013). The study was exempt by the institutional review board and the informed consent, due to the retrospective nature of the study and the establishment of a previous protocol for conducting retrospective studies.

\section{Radiation therapy}

For treatment, patients were immobilized using a fullbody vacuum bag system for position stabilization and consistency. Serial computed tomography (CT) scans were taken (free-breathing, inhale, exhale) for treatment planning purposes to assess the motion of the target during the breath cycle and generate an internal-targetvolume (ITV) margin around the GTV. For patients with previous radiation therapy, treatment planning files were obtained and imported into the treatment planning system for evaluation of prior dose to organs-at-risk. Dose was prescribed to the planning target volume (PTV), which was defined as the ITV plus 3-5 millimeters of margin to account for uncertainties in imaging and localization. In general, a 3D conformal treatment planning approach with non-coplanar gantry angles was used to minimize dosimetric overlap of entrance and exit portals. SBRT was delivered using a $6 \mathrm{MV}$ photon beam on a linear accelerator with a $2.5-4 \mathrm{~mm}$ width multi-leaf collimator for custom shaping of portals. On-board cone-beam CT (CBCT) with 4D capabilities was used prior to treatment and would be repeated during treatment as needed to correct any intrafraction motion of the patient or target. Treatment was generally provided once or twice weekly, per institutional policy.

For early stage tumors, the most common treatment regimens included 60 Gy in 4 fractions [ $\mathrm{n}=17$, biologically effective dose $(\mathrm{BED})=150 \mathrm{~Gy}$ ], 50 Gy in 4 fractions $(\mathrm{n}=28$, BED $=112.5$ Gy), and 50 Gy in 5 fractions $(n=13$, BED $=100 \mathrm{~Gy}) .40$ Gy in 4 fractions $(\mathrm{n}=12, \mathrm{BED}=80 \mathrm{~Gy})$ was commonly chosen in the setting of prior radiation therapy. Nodal targets often received lower doses, due to concern for potential toxicity. These most commonly included $28 \mathrm{~Gy}$ in 4 fractions $(\mathrm{n}=8, \mathrm{BED}=47.6 \mathrm{~Gy}), 24 \mathrm{~Gy}$ in 4 fractions $(\mathrm{n}=8$, BED $=38.4$ ), or $20 \mathrm{~Gy}$ in 4 fractions $(\mathrm{n}=7, \mathrm{BED}=30 \mathrm{~Gy})$. Other dose-fractionation schemes for nodal targets included
36 Gy in 4 fractions or 45 Gy in 3 fractions. In 11 cases, prescription doses of at least 50 Gy were delivered. This was generally done in the setting of minimal prior treatment and treatments for the hilar lymph nodes (as opposed to other mediastinal targets). Lower dose regimens, such as 15-21 Gy in 3 fractions $(n=6)$, were sometimes utilized, especially in the setting of reirradiation. Most treatments involved 3-5 fractions of therapy, with only two treatments involving one or two fractions. The one case in which only one treatment was offered was included because it contributed to the analysis of treatments involving prior thoracic surgery. Biologically effective dose (BED) was calculated using the formula dose $\times(1+$ dose per fraction/alpha beta ratio), and an alpha beta ratio of 10 Gy was used.

SBRT is not considered the standard of care for advanced or recurrent disease. Patients treated with such disease with SBRT in this study generally were considered to be highrisk candidates for whom concurrent chemoradiation to $60 \mathrm{~Gy}$ was considered to be a suboptimal choice. Many of these individuals had received prior treatment or declined these more standard treatment approaches, due to concerns regarding toxicity. Due to these constraints, relatively lower treatment doses were often used, as well. In the setting of these nodal treatments, dose constraints varied, as dictated by the impact of prior radiation therapy fields. Generally, however, a maximum point dose of $105 \%$ of the prescription dose was allowed to the esophagus and proximal tracheobronchial tree. Whenever possible, these maximal doses were kept to less than 30 Gy.

Key dosimetric characteristics of the treatment were recorded, including dose, fractions, days between fractions, and PTV. The use of concurrent chemotherapy was noted, where applicable. The reason for SBRT was recorded, as were any previous therapies: chemotherapy, thoracic surgery, and thoracic radiotherapy. Synchronous lesions were defined as two or more lesions present in a patient's lungs. This included two lesions within the same lobe, different lobes, or different lungs. The presence of synchronous lesions, as well as the target (nodal vs. nonnodal), were considered as potential predictive factors for local control in the multivariate analysis. Since many of these patients were treated prior to the emergence of data concerning the benefits of immunotherapy, this data was not recorded.

\section{Patient outcomes}

The principal outcomes of the study were local control, 
overall survival, progression-free survival, and localprogression free survival. These were assessed via clinical and imaging follow-up, the latter of which was generally conducted 2-3 months after completion of SBRT. The imaging modality used for this follow-up was recorded, generally consisting of CT and positron emission tomography (PET) scans. Tumor recurrence was recorded as the date of the imaging which noted the recurrence, and it was designated as local, regional, or distant failure. Local failure was defined as tumor recurrence within the same lobe as the primary tumor or in the ipsilateral hilum. Regional failure was defined as tumor recurrence in the contralateral hilum or ipsilateral lung but a different lobe than the primary tumor. Other cases of recurrence were deemed to be distant failure. Local control was still evaluated even after the detection of regional of distant metastases. Similarly, regional control was still assessed even after the detection of distant metastases, as opposed to censoring at the time of distant failure. Overall survival was defined as the time between the last day of treatment and either the date of death (if known) or the last date of interaction with the medical system. Toxicity was recorded and graded according to the Common Terminology Criteria for Adverse Events v4.0. Toxicity was most commonly reported on subsequent follow-up visits with radiation oncology, but pulmonology follow-up also contributed to the toxicity outcomes.

\section{Statistical methods}

Statistical analysis involved univariate analysis, Cox proportional-hazards analysis, and the Kaplan-Meier method. A P value of 0.05 was considered to be the threshold for statistical significance. Univariate analyses involved exclusively two-tailed $t$-tests and served to compare binary variables. Cox proportional-hazards analysis demonstrated hazard ratios for the potential predictive factors. Though all independent variables were initially assessed, very low variance was detected for non-surgical candidacy for the overall survival analysis, so the reason for SBRT was eliminated from the subsequent hazard analysis for overall survival. The Kaplan-Meier method was used to portray time-dependent variables, and stratifications of the data were conducted according to the results of the initial statistical analyses. Treatments for stage 3 disease were analyzed as an important subgroup of the analysis, and analysis was conducted using the statistical methods previously described. Any patients or treatments with missing data for a particular statistical analysis were not included in that analysis.

\section{Results}

\section{Patient characteristics}

A total of 115 unique patients with 148 treated lesions were included (Table 1). Patients started treatment at a median 78 (53.1-92.2) years of age, and 60 of the 115 patients had chronic obstructive pulmonary disease (COPD). Patients presented at a median $9.8(0.4-233.0)$ months after primary diagnosis, and only 56 (49\%) obtained biopsy prior to SBRT. $93 \%$ of treated lesions had controlled systemic disease, with a median longest dimension of $2(1-6.7) \mathrm{cm}$. Lesions were treated at clinical stage 1 (46\%), 2 (20\%), 3 (28\%), 4 (4\%), and unknown (1\%). 58 treatments involved nodal targets. Synchronous lesions were present in $31 \%$ of cases, and $75 \%$ had a pre-treatment PET scan, demonstrating a median pre-treatment standardized uptake value (SUV) of 4.5 (0-17.1).

\section{Radiotherapy and prior treatments}

Patients were treated with a median 45 [5-60] Gy in 4 [1-5] fractions. The median days between fractions was $5.3[0-18]$, and the median overall treatment time was 21 days. Concurrent chemotherapy was given with only $3 \%$ of treatments. The median PTV of treatments was 25.7 (1.3-269.6) cc. SBRT was offered for primary cancer (39\%), recurrence $(57 \%)$, and medically inoperable status $(5 \%)$. Many treatments involved lesions that were previously treated with chemotherapy (32\%), thoracic surgery (21\%), and thoracic radiotherapy (49\%). Of these prior radiation treatments, the median radiation dose was 57 Gy in 5 fractions, and these treatments occurred at a median 15.6 months prior to SBRT.

\section{Patient outcomes}

Median overall survival, progression-free survival, and local-progression free survival were 20.0, 14.0, and 16.1 months, respectively. There were 28 instances of local failure $(19 \%)$ at a median imaging follow-up of 13.2 months. Median clinical follow-up was 17.5 months. There were 20 cases of regional failure (14\%), which occurred at a median 6.16 months. Of these, 11 occurred in patients with stage 3 disease, as opposed to only 3 and 
Table 1 Patient demographics and treatment characteristics are considered

\begin{tabular}{|c|c|}
\hline Variable & Number \\
\hline \multicolumn{2}{|l|}{ Patient demographics } \\
\hline Patients & 115 \\
\hline Lesions & 148 \\
\hline Median age (years) & 78 (53.0-92) \\
\hline Patients with COPD & $60(52 \%)$ \\
\hline $\begin{array}{l}\text { Median time from primary diagnosis to SBRT } \\
\text { (months) }\end{array}$ & $9.8(0.4-233)$ \\
\hline Patients with biopsy obtained & $56(49 \%)$ \\
\hline Patients without biopsy obtained & $59(51 \%)$ \\
\hline Squamous cell carcinoma & $25(17 \%)$ \\
\hline Adenocarcinoma & $32(22 \%)$ \\
\hline Other or unknown histology & $91(61 \%)$ \\
\hline Controlled systemic disease & $137(93 \%)$ \\
\hline Lesions with pre-treatment PET & $111(75 \%)$ \\
\hline Median pre-treatment SUV & $4.5(0-17.1)$ \\
\hline${ }^{\star}$ Median longest dimension (cm) & $2(1-6.7)$ \\
\hline Stage 1 & $68(46 \%)$ \\
\hline Stage 2 & $30(20 \%)$ \\
\hline Stage 3 & $42(28 \%)$ \\
\hline Stage 4 & $6(4 \%)$ \\
\hline Treatment history or characteristic & Number \\
\hline Median dose (Gy) & $45(5.0-60)$ \\
\hline Median fractions & $4(1.0-5)$ \\
\hline Median BED (Gy) & $100(7.5-180)$ \\
\hline Median days between fractions & $5(0-18)$ \\
\hline Concurrent chemotherapy & $4(3 \%)$ \\
\hline Median PTV (cc) & $25.7(1.28-69.6)$ \\
\hline Reason for SBRT: primary cancer & 57 (39\%) \\
\hline Reason for SBRT: recurrence & $84(57 \%)$ \\
\hline Reason for SBRT: not surgical candidate & $7(5 \%)$ \\
\hline Treatments with prior chemotherapy & $48(32 \%)$ \\
\hline Treatments with prior surgery & $31(21 \%)$ \\
\hline Treatments with prior thoracic radiotherapy & $72(49 \%)$ \\
\hline Median prior radiotherapy dose (Gy) & 57 \\
\hline Median prior radiotherapy fractions & 5 \\
\hline
\end{tabular}

Table 1 (continued)
Table 1 (continued)

\begin{tabular}{lc}
\hline Variable & Number \\
\hline $\begin{array}{l}\text { Median time from prior radiotherapy to SBRT } \\
\text { (months) }\end{array}$ & $15.6(0.2-281)$ \\
Synchronous lesions & $46(31 \%)$ \\
Nodal target & $58(39 \%)$ \\
Central, non-nodal target & $90(61 \%)$ \\
\hline
\end{tabular}

*This includes nodes and primary disease. COPD, chronic obstructive pulmonary disease; SBRT, stereotactic body radiation therapy; PET, positron emission tomography; SUV, standardized uptake value; BED, biologically effective dose; PTV, planning target volume.

6 cases for stage 2 and stage 1 patients, respectively. 15 distant failures $(10 \%)$ were noted at a median 9.3 months. Fifty lesions (34\%) demonstrated some disease progression (local, regional, or distant). Using the KaplanMeier method, the 2 -year overall survival was $65 \%$. Twoyear progression free survival and local-progression free survival were $48 \%$ and $53 \%$, respectively. Two-year local control was $77 \%$ (Table 2).

\section{Predictive factors}

On univariate analysis, BED >100 Gy demonstrated a trend towards improved local control $(\mathrm{P}=0.10)$. Higher clinical stage trended towards increased disease progression $(\mathrm{P}=0.051)$, and prior chemotherapy was predictive of this outcome $(\mathrm{P}=0.017)$.

Cox proportional-hazards analysis was performed, showing that prior chemotherapy trended towards increased local failure $(\mathrm{P}=0.05)$, with a hazard ratio of $2.49(0.98-$ 6.31). Disease progression had multiple predictive factors (Figure 1). Synchronous lesions $(\mathrm{P}=0.03)$ and prior radiotherapy $(\mathrm{P}=0.03)$ were protective, with hazard ratios of 0.35 (0.13-0.92) and 0.25 (0.07-0.89), respectively. Prior chemotherapy $(\mathrm{P}=0.04)$ and prior thoracic surgery $(\mathrm{P}=0.05)$ demonstrated increased disease progression, with hazard ratios of 2.44 (1.02-5.82) and $2.36(1.00-5.58)$. Finally, prior thoracic surgery predicted for decreased overall survival $(\mathrm{P}<0.005)$, with a hazard ratio of 4.39 (2.10-9.20). There was also a trend towards improved overall survival with obtaining a biopsy $(\mathrm{P}=0.08)$ while prior chemotherapy trended towards decreased overall survival $(\mathrm{P}=0.11)$.

Kaplan-Meier analysis was utilized to demonstrate local 
Table 2 Key patient outcomes are tabulated

\begin{tabular}{lcc}
\hline Variable & Number & Rate \\
\hline 2-year overall survival & $65 \%$ \\
2-year progression-free survival & $48 \%$ \\
2-year local-progression free survival & $53 \%$ \\
2-year local control & $77 \%$ \\
Local failures & 28 & $19 \%$ \\
Median time to local failure (months) & 15.2 & \\
Regional failure & 20 & $14 \%$ \\
Median time to regional failure (months) & 6.2 & \\
Distant failure & 15 & $10 \%$ \\
Median time to distant failure (months) & 9.3 & \\
Any disease progression & 50 & $34 \%$ \\
Grade 1 toxicity & 13 & $9 \%$ \\
Grade 2 toxicity & 14 & $9 \%$ \\
Grade $\geq 3$ toxicity & 0 & $0 \%$ \\
Median imaging follow-up (months) & 13.2 & \\
Median clinical follow-up (months) & 17.5 & \\
\hline & & \\
\end{tabular}

control, local-progression free survival, progression-free survival, and overall survival for the entire cohort (Figure 2). It was demonstrated that nodal targets had worse localprogression free survival than non-nodal targets $(\mathrm{P}=0.05)$. Patients without prior chemotherapy had improved progression-free survival $(\mathrm{P}=0.11)$ and trended towards improved overall survival $(\mathrm{P}=0.05)$. Similarly, patients without prior thoracic surgery had improved progressionfree survival $(\mathrm{P}<0.005)$ and overall survival $(\mathrm{P}<0.005)$.

\section{Stage 3 disease}

Patients with stage 3 disease were also considered separately as part of the outcomes analysis to compare their outcomes to patients with early stage disease. Two patients had a clinical stage unavailable, and two patients (six treated lesions) were also not considered because they had stage 4 disease. A total of 111 patients with 140 treatments were, therefore, used for this subgroup analysis. The stage 3 group consisted of 27 patients and 42 treated lesions. Of these, only 1 treatment involved concurrent chemotherapy, but most treatments involved prior chemotherapy (55\%), thoracic surgery $(31 \%)$, or radiation $(76 \%)$. Forty-three percent of treatments involved patients with COPD. The 2 -year local control for stage 3 disease was $82 \%$, and the 2 -year overall survival was $59 \%$. There was no difference between stage 3 disease and early stage disease for 2 -year local control (82\% vs. 77\%, respectively); however, stage 3 disease trended towards decreased overall survival and progression-free survival $(\mathrm{P}=0.18$ and $\mathrm{P}=0.32$, respectively) (Figure 3).

\section{Toxicity}

There were 13 total acute and late grade 1 toxicities reported (9\%), in addition to 14 total grade 2 toxicities (9\%). There were no reported grade $\geq 3$ morbidities and no grade 5 toxicities. The most common grade 1 side effect was fatigue (7 cases); others included dyspnea, cough, shortness of breath, nausea, and esophagitis. The most common grade 2 toxicities were pneumonitis and shortness of breath (3 cases each). The other grade 2 morbidities involved fatigue, dyspnea, cough, pleural effusion, pain, and one rib fracture. Though attempts were made to risk stratify the patients for increased rates of toxicity based on various disease characteristics, the relatively low number of overall side effects prevented a rigorous analysis of this kind.

\section{Discussion}

Overall, this study demonstrated encouraging treatment outcomes in a moderately sized cohort of centrally-located NSCLC treated with SBRT. Despite a high rate of adverse risk factors in this data set, an impressive 2-year local control of $77 \%$ was reported. Patients were treated with an increased inter-fraction time, likely contributing to the $0 \%$ rate of grade $\geq 3$ toxicity.

A six-year analysis of 102 primary lung cancers treated with once-weekly SBRT from Salazar et al. is perhaps the most comparable study to the present analysis (21). The study included 30 stage 3 cancers, and the authors documented $12 \%, 7 \%$, and $0 \%$ rates of grade 1,2 , and $\geq 3$ toxicity, respectively. These results are similar to those of the present analysis and point towards the potential utility of SBRT in stage 3 disease, especially in elderly patients or those who are poor candidates for combined chemotherapy and radiation. Further, it adds support to the hypothesis that increased inter-fraction time can help to minimize treatment toxicity. Rates of symptomatic radiation pneumonitis with SBRT have ranged from 9\% to $28 \%$, but here only 3 cases (2\%) were found, despite high rates 


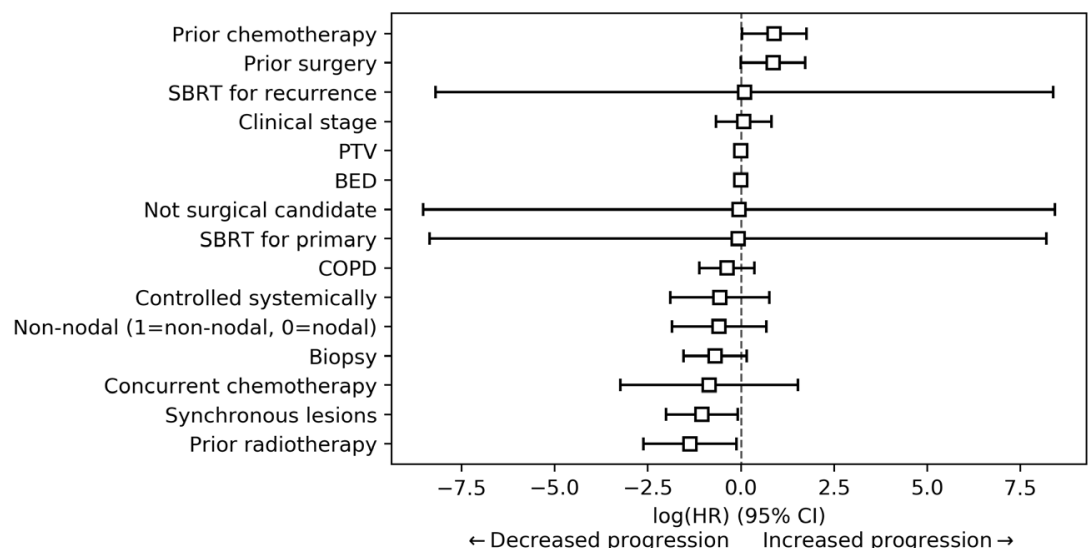

Figure 1 Cox proportional-hazards analysis demonstrates predictive factors for disease progression.
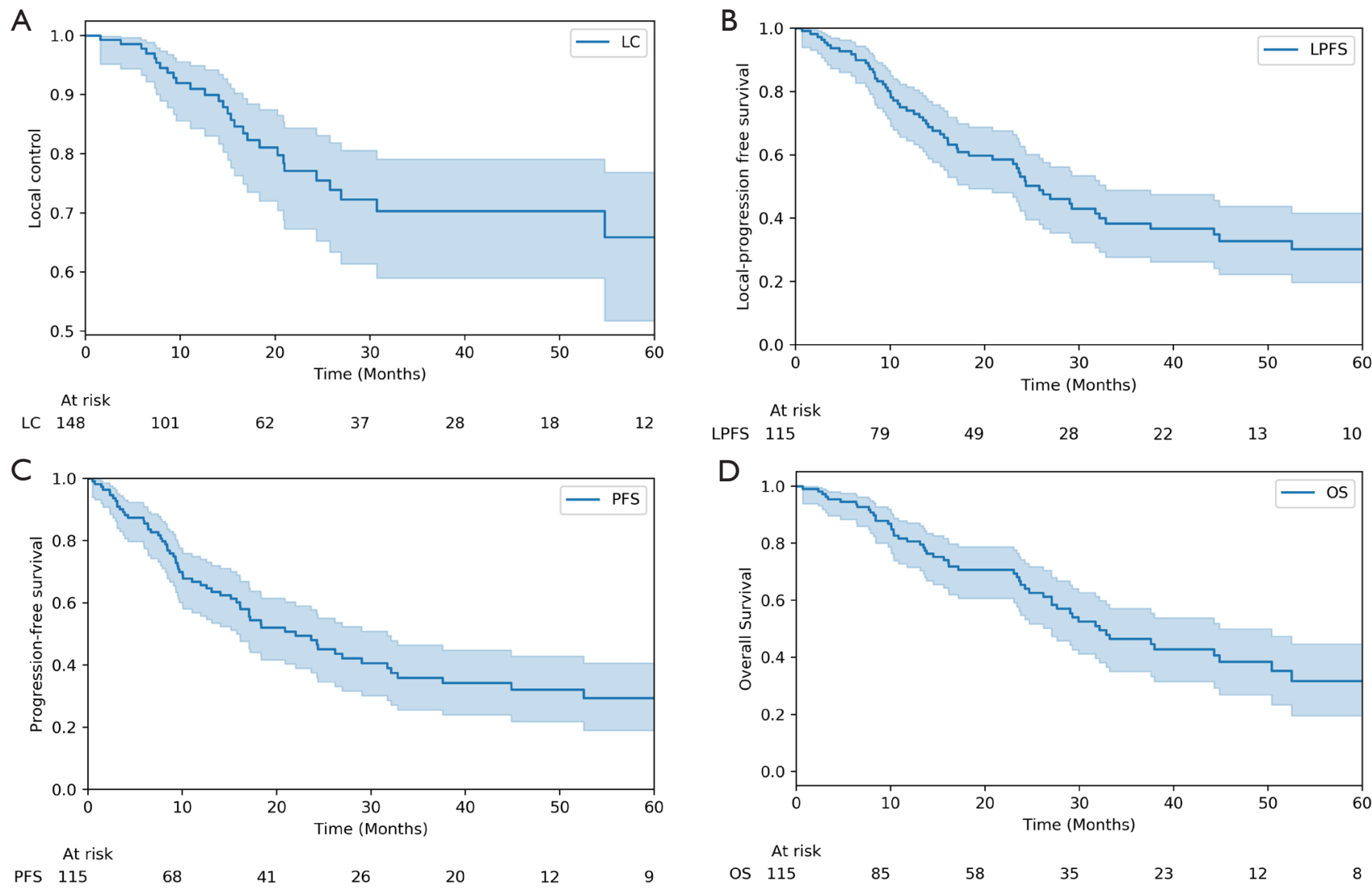

Figure 2 Kaplan-Meier analysis was utilized to demonstrate (A) local control, (B) local-progression free survival (LPFS), (C) progressionfree survival (PFS), and (D) overall survival (OS).

of adverse risk factors $(5,22)$. In addition, patients have commented on the flexibility of once-weekly treatments because weekly activities can be continued relatively uninterrupted by radiation therapy. This treatment strategy may be particularly optimal for patients living relatively near the treatment facility while patients who traveled a long distance for treatment may prefer to complete therapy sooner. 
A
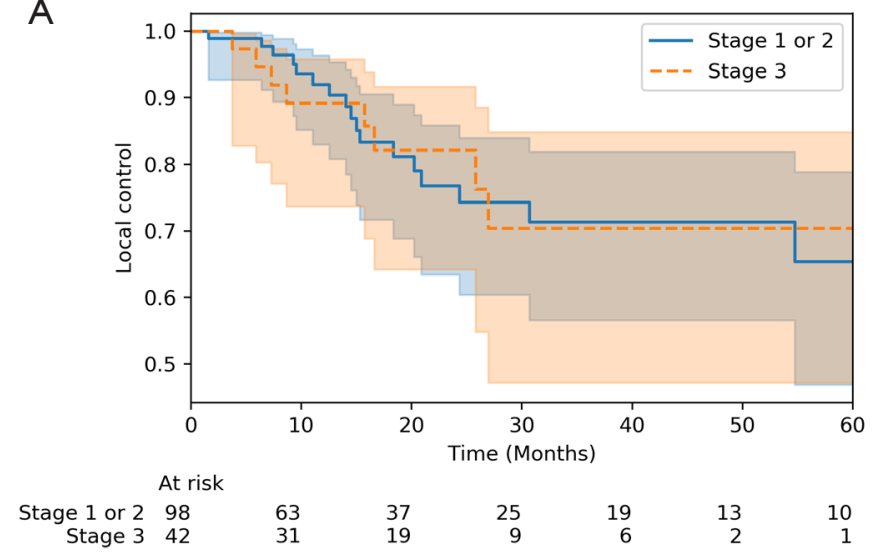

B

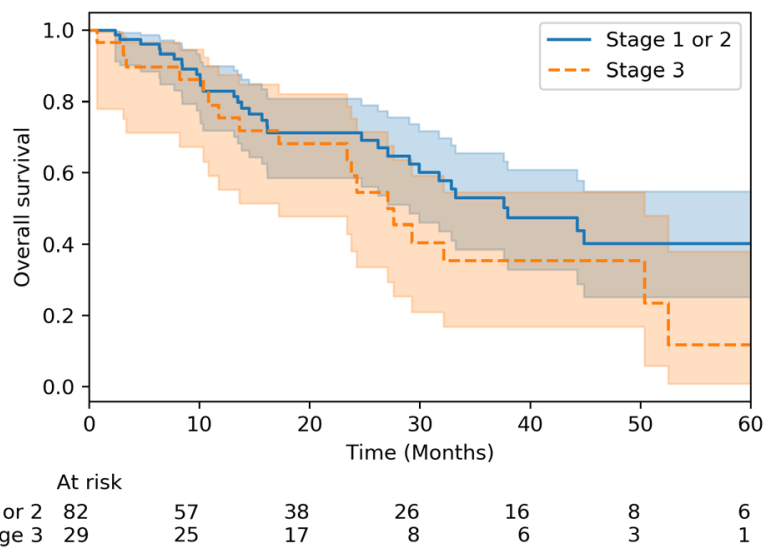

Figure 3 SBRT for stage 3 disease demonstrated comparable local control (LC) to treatments for stage 1 or 2 disease (A, P=0.94), but patients with stage 3 disease trended towards decreased overall survival (OS) (B, $\mathrm{P}=0.18)$. SBRT, stereotactic body radiation therapy.

This analysis consisted of a unique patient cohort with more advanced disease and adverse risk factors than most lung SBRT studies. Generally, most lung SBRT has focused on early stage NSCLC or oligometastatic disease, but this study was quite novel in that 42 treatments for stage $\geq 3$ NSCLC were included $(1,23,24)$. SBRT has been used as a method to boost residual disease in locally advanced NSCLC, but rarely has it been considered for definitive therapy (19). Despite trends towards decreased overall and progression-free survival for stage 3 disease, this study demonstrated comparable local control rates for stage 3 disease compared to early stage NSCLC. Such a result is hypothesis-generating and presents the possibility of treating stage 3 disease with SBRT for palliation or local control.

Most treatments for stage 3 disease were the result of $\mathrm{N} 2$ or $\mathrm{N} 3$ disease, rather than $\mathrm{T} 3 \mathrm{~N} 1$ or $\mathrm{T} 4$ tumors, and most dose-fractionation schemes involved the delivery of 20-30 Gy in 4 fractions. These treatments resulted in an encouraging side effect profile that was not significantly different than that for other targets. It is likely that the increased inter-fraction time (and potentially the lower treatment doses) used in this analysis contributed to the $0 \%$ rate of grade $\geq 3$ toxicity. These outcomes for locallyadvanced NSCLC indicate that SBRT may prove beneficial for carefully selected patients (particularly with N2 or N3 disease, rather than large tumor size). Further study is recommended to assess for the role of increased interfraction time in this setting as a potential tool to reduce toxicity in high-risk patients.
The underlying radiobiology of SBRT involves key differences from that of conventional radiotherapy, and it remains unclear whether classic radiobiologic modeling and the linear-quadratic model appropriately describe cell death in this clinical scenario $(25,26)$. Preclinical and modeling studies have indicated that hypoxia may have a particularly negative impact on tumor control in SBRT, and the time between fractions in conventional radiotherapy allows for improved reoxygenation (26). Another study of SBRT in murine tumors indicated that the surviving hypoxic cells decreased over several days post irradiation, likely secondary to reoxygenation (27). The authors hypothesized that SBRT may be significantly improved by allowing an inter-fraction time for reoxygenation while avoiding repopulation, which occurred after reoxygenation. Finally, high doses of radiation therapy may serve to slow cell cycle progression, and it is possible that increasing inter-fraction time may serve to allow for increased redistribution within the cell cycle and therefore additional tumor cell killing (28). It appears to be biologically plausible for an increased interfraction time to reduce toxicity, but prospective studies would be needed to confirm this hypothesis-generating result.

Several important risk stratifications were identified for central lung SBRT. Though BED >100 Gy trended towards improved local control in support of prior literature, lower dose treatments were also effective (1). Patients who obtained a biopsy trended towards improved overall survival. This may merely be the result of patients with fewer medical comorbidities having an increasing likelihood 
of obtaining a biopsy, and these patients have improved overall survival, irrespective of their lung cancer. Intuitively, clinical stage, prior chemotherapy, and prior thoracic surgery trended towards increased disease progression, as these factors related to advanced or recurrent disease (21). In the case of prior thoracic surgery, patients presenting with recurrent disease may represent a different clinical scenario for SBRT than patients presenting with primary disease (29). While nodal targets trended towards decreased local-progression free survival, the fact that this variable was not predictive of overall survival or progression-free survival suggests that therapy offered clinical benefit (16). Prior radiotherapy was interestingly predictive of decreased disease progression. This result supports offering repeat courses of SBRT after recurrence. $57 \%$ of the treatments in this cohort involved recurrent cancer, and $49 \%$ of treatments had prior thoracic radiotherapy, offering a fairly large patient group to support such a hypothesis. Parks et al. studied a set of 27 patients treated with prior thoracic radiation for stage IIB-IIIB NSCLC and demonstrated encouraging outcomes but high rates of toxicity (63\% rate of symptomatic pneumonitis) (30). While it is possible that incorporating increased inter-fraction time could serve to improve toxicity outcomes in cases of reirradiation, further study is needed. The prior courses of radiotherapy included in this cohort were heterogenous, including other courses of SBRT as well as concurrent chemoradiation to 60 Gy in 30 fractions. These significant differences indicate that these findings concerning reirradiation should be considered with caution.

The key limitation of this study is the variation in dosefractionation schemes used for the different tumors. While this can partly be explained by the range of clinical stages included in the analysis, comparing these treatments presents inherent difficulties. Prospective analysis could select more specific dose-fractionation schemes for the different stages. Additional information to more completely describe the nodal distribution of the nodal targets in this study would have also proven beneficial. A prospective study could expand upon the hypothesis-generating results presented here and consider a cohort of patient treated with a more homogeneous inter-fraction time (e.g., exactly one week). Further, some of the variables considered may be interdependent, such as increasing stage with prior chemotherapy and nodal targets. Finally, multi-institutional study may serve to determine patients at higher risk for toxicity with the once-weekly fractionation. Because the rates of side effects were so low using this treatment regimen, a large patient cohort may be required for such an analysis.

\section{Conclusions}

Our series demonstrated encouraging local control with low rates of toxicity for central lung SBRT, including many patients with stage 3 disease. This may be the result of the relatively large inter-fraction interval. This interval may allow for greater tumor effects (such as reoxygenation) and improved tolerance from normal tissues.

\section{Acknowledgments}

Funding: None.

\section{Footnote}

Reporting Checklist: The authors have completed the STROBE reporting checklist. Available at http://dx.doi. org/10.21037/jtd-20-2659

Data Sharing Statement: Available at http://dx.doi. org/10.21037/jtd-20-2659

Peer Review File: Available at http://dx.doi.org/10.21037/jtd20-2659

Conflicts of Interest: All authors have completed the ICMJE uniform disclosure form (available at http://dx.doi. org/10.21037/jtd-20-2659). ROK reports that his wife is a senior technical product manager at GE Healthcare. The other authors have no conflicts of interest to declare.

Ethical Statement: The authors are accountable for all aspects of the work in ensuring that questions related to the accuracy or integrity of any part of the work are appropriately investigated and resolved. This study was conducted in accordance with the Declaration of Helsinki (as revised in 2013). The study was exempt by the institutional review board and the informed consent, due to the retrospective nature of the study and the establishment of a previous protocol for conducting retrospective studies.

Open Access Statement: This is an Open Access article distributed in accordance with the Creative Commons Attribution-NonCommercial-NoDerivs 4.0 International License (CC BY-NC-ND 4.0), which permits the non- 
commercial replication and distribution of the article with the strict proviso that no changes or edits are made and the original work is properly cited (including links to both the formal publication through the relevant DOI and the license). See: https://creativecommons.org/licenses/by-nc-nd/4.0/.

\section{References}

1. Grills IS, Hope AJ, Guckenberger M, et al. A collaborative analysis of stereotactic lung radiotherapy outcomes for early-stage non-small-cell lung cancer using daily online cone-beam computed tomography image-guided radiotherapy. J Thorac Oncol 2012;7:1382-93.

2. Moghanaki D, Chang JY. Is surgery still the optimal treatment for stage I non-small cell lung cancer? Transl Lung Cancer Res 2016;5:183.

3. Palma D, Senan S. Stereotactic radiation therapy: changing treatment paradigms for stage I nonsmall cell lung cancer. Curr Opin Oncol 2011;23:133-9.

4. Ball D, Mai GT, Vinod S, et al. Stereotactic ablative radiotherapy versus standard radiotherapy in stage 1 nonsmall-cell lung cancer (TROG 09.02 CHISEL): a phase 3 , open-label, randomised controlled trial. Lancet Oncol 2019;20:494-503.

5. Yamashita H, Takahashi W, Haga A, et al. Radiation pneumonitis after stereotactic radiation therapy for lung cancer. World J Radiol 2014;6:708.

6. Chang JY, Bezjak A, Mornex F, et al. Stereotactic ablative radiotherapy for centrally located early stage non-smallcell lung cancer: what we have learned. J Thorac Oncol 2015;10:577-85.

7. Bezjak A, Paulus R, Gaspar LE, et al. Safety and efficacy of a five-fraction stereotactic body radiotherapy schedule for centrally located non-small-cell lung cancer: NRG Oncology/RTOG 0813 trial. J Clin Oncol 2019;37:1316-25.

8. Senthi S, Haasbeek CJ, Slotman BJ, et al. Outcomes of stereotactic ablative radiotherapy for central lung tumours: a systematic review. Radiother Oncol 2013;106:276-82.

9. Bral S, Gevaert T, Linthout N, et al. Prospective, riskadapted strategy of stereotactic body radiotherapy for early-stage non-small-cell lung cancer: results of a phase II trial. Int J Radiat Oncol Biol Phys 2011;80:1343-9.

10. Rowe BP, Boffa DJ, Wilson LD, et al. Stereotactic body radiotherapy for central lung tumors. J Thorac Oncol 2012;7:1394-9.

11. Tekatli H, Senan S, Dahele M, et al. Stereotactic ablative radiotherapy (SABR) for central lung tumors: plan quality and long-term clinical outcomes. Radiother Oncol 2015;117:64-70.

12. Haasbeek CJ, Lagerwaard FJ, Slotman BJ, et al. Outcomes of stereotactic ablative radiotherapy for centrally located early-stage lung cancer. J Thorac Oncol 2011;6:2036-43.

13. Chang JH, Poon I, Erler D, et al. The safety and effectiveness of stereotactic body radiotherapy for central versus ultracentral lung tumors. Radiother Oncol 2018;129:277-83.

14. Murrell DH, Laba JM, Erickson A, et al. Stereotactic ablative radiotherapy for ultra-central lung tumors: prioritize target coverage or organs at risk? Radiat Oncol 2018;13:57.

15. Nguyen KN, Hause DJ, Novak J, et al. Tumor control and toxicity after SBRT for ultracentral, central, and paramediastinal lung tumors. Pract Radiat Oncol 2019;9:e196-202.

16. Horne ZD, Richman AH, Dohopolski MJ, et al. Stereotactic body radiation therapy for isolated hilar and mediastinal non-small cell lung cancers. Lung Cancer 2018;115:1-4.

17. Oshiro Y, Aruga T, Tsuboi K, et al. Stereotactic body radiotherapy for lung tumors at the pulmonary hilum. Strahlenther Onkol 2010;186:274-9.

18. Manabe Y, Shibamoto Y, Baba F, et al. Definitive radiotherapy for hilar and/or mediastinal lymph node metastases after stereotactic body radiotherapy or surgery for stage I non-small cell lung cancer: 5-year results. Jpn J Radiol 2018;36:719-25.

19. Feddock J, Arnold SM, Shelton BJ, et al. Stereotactic body radiation therapy can be used safely to boost residual disease in locally advanced non-small cell lung cancer: a prospective study. Int J Radiat Oncol Biol Phys 2013;85:1325-31.

20. Quon HC, Ong A, Cheung P, et al. Once-weekly versus every-other-day stereotactic body radiotherapy in patients with prostate cancer (PATRIOT): A phase 2 randomized trial. Radiother Oncol 2018;127:206-12.

21. Salazar OM, Sandhu TS, Lattin PB, et al. Once-weekly, high-dose stereotactic body radiotherapy for lung cancer: 6-year analysis of 60 early-stage, 42 locally advanced, and 7 metastatic lung cancers. Int J Radiat Oncol Biol Phys 2008;72:707-15.

22. Takeda A, Kunieda E, Ohashi T, et al. Severe COPD is correlated with mild radiation pneumonitis following stereotactic body radiotherapy. Chest 2012;141:858-66.

23. Okunieff P, Petersen AL, Philip A, et al. Stereotactic body radiation therapy (SBRT) for lung metastases. Acta Oncol 
2006;45:808-17.

24. Aoki M, Hatayama Y, Kawaguchi H, et al. Stereotactic body radiotherapy for lung metastases as oligo-recurrence: a single institutional study. J Radiat Res 2016;57:55-61.

25. Macià I Garau M. Radiobiology of stereotactic body radiation therapy (SBRT). Rep Pract Oncol Radiother 2017;22:86-95.

26. Brown JM, Carlson DJ, Brenner DJ. The tumor radiobiology of SRS and SBRT: are more than the 5 Rs involved? Int J Radiat Oncol Biol Phys 2014;88:254-62.

27. Song CW, Griffin RJ, Lee YJ, et al. Reoxygenation and Repopulation of Tumor Cells after Ablative Hypofractionated Radiotherapy (SBRT and SRS) in

Cite this article as: Kowalchuk RO, Waters MR, Richardson KM, Spencer KM, Larner JM, Kersh CR. A single institutional experience with central lung stereotactic body radiation therapy demonstrating encouraging results with increased inter-fraction time. J Thorac Dis 2021;13(2):642-652. doi: 10.21037/jtd-202659
Murine Tumors. Radiat Res 2019;192:159-68.

28. Kim MS, Kim W, Park IH, et al. Radiobiological mechanisms of stereotactic body radiation therapy and stereotactic radiation surgery. Radiat Oncol J 2015;33:265.

29. Kowalchuk RO, Waters MR, Richardson KM, et al. Stereotactic body radiation therapy for salvage treatment of recurrent non-small cell lung cancer. Pract Radiat Oncol 2020;10:e475-84.

30. Parks J, Kloecker G, Woo S, et al. Stereotactic body radiation therapy as salvage for intrathoracic recurrence in patients with previously irradiated locally advanced nonsmall cell lung cancer. Am J Clin Oncol 2016;39:147-53. 